\title{
Singlet and non-singlet axial-vector form factors for the octet baryons.
}

\author{
Harleen Dahiya ${ }^{* \dagger}$ \\ Department of Physics, Dr. B.R. Ambedkar National Institute of Technology, Jalandhar, 144011, \\ India \\ E-mail: dahiyah@nitj.ac.in
}

\section{Monika Randhawa}

University Institute of Engineering and Technology, Panjab University, Chandigarh, 160014, India

We have studied the implications of chiral symmetry breaking and SU(3) symmetry breaking for the axial-vector form factors of the low lying octet baryons $(N, \Sigma, \Xi$ and $\Lambda)$ in the chiral constituent quark model $(\chi \mathrm{CQM})$. We have calculated the singlet $\left(g_{A}^{0}\right)$ and non-singlet $\left(g_{A}^{3}\right.$ and $g_{A}^{8}$ ) axial-vector coupling constants expressed as combinations of the spin polarizations at zero momentum transfer. We have used the conventional dipole form of parametrization to analyse the $Q^{2}$ dependence of the axial-vector form factors $\left(G_{A}^{0}\left(Q^{2}\right), G_{A}^{3}\left(Q^{2}\right)\right.$ and $\left.G_{A}^{8}\left(Q^{2}\right)\right)$.

XXIII International Workshop on Deep-Inelastic Scattering

27 April - May 12015

Dallas, Texas

\footnotetext{
* Speaker.

${ }^{\dagger}$ Authors would like to thank Department of Science and Technology, Government of India for financial support.
} 


\section{Introduction}

The internal structure of the baryons has been extensively studied ever since the measurements of polarized structure functions of proton in the deep inelastic scattering (DIS) experiments $[1,2,3]$. These experiments have provided the first evidence that the valence quarks of proton carry only a small fraction of its spin and the decomposition of the proton's spin still remains to be a major unresolved issue in high energy spin physics. Form factors parameterized from the electromagnetic current operator as well as the isovector axial-vector current operator are important in hadron physics as they provide a deep insight in understanding the internal structure. The electromagnetic Dirac and Pauli form factors are well know over a wide region of momentum transfer squared $Q^{2}$, however, the study of the axial-vector form factors has been rather limited. Recently, experiments measuring electromagnetic and weak form factors from the elastic scattering of electrons, for example, SAMPLE at MIT-Bates [4], G0 at JLab [5], PVA4 at MAMI [6] and HAPPEX at JLab [7] have given indications of strangeness contribution in the nucleon. These experiments have provided considerable insight on the role played by strange quarks in the charge, current and spin structure of the nucleon. The nucleon axial coupling constant $g_{A}^{3}$ has received much attention in the past and has been determined precisely from nuclear $\beta$-decay [8]. Even though there has been considerable progress in the past few years to determine the $Q^{2}$ dependence of axial form factors experimentally, there is no consensus regarding the various mechanisms which can contribute to it. Experiments involving elastic scattering of neutrinos and antineutrinos [9] and the pion electro-production on the proton [10] have explored $Q^{2}$ dependence of axial form factors in the past and they point out the need for additional refined data.

The broader question of axial charge, axial form factors and the strange quark contribution to the axial form factors of the nucleon has also been discussed by several authors in other models recently [11]. One of the most successful nonperturbative approach which finds its application for the quantities discussed above is the chiral constituent quark model ( $\chi \mathrm{CQM})$ [12]. The $\chi \mathrm{CQM}$ successfully explains the "proton spin problem", magnetic moments of octet and decuplet baryons including their transitions [13], account for the violation of Gottfried Sum Rule [14], hyperon $\beta$ decay parameters [15], strangeness content in the nucleon [16], magnetic moments of $\frac{1}{2}^{-}$octet baryon resonances [17], magnetic moments of $\frac{1}{2}^{-}$and $\frac{3}{2}^{-} \Lambda$ resonances [18], etc.. The model is successfully extended to predict the important role played by the small intrinsic charm content in the nucleon spin in the SU(4) $\chi \mathrm{CQM}[19,20,21]$.

\section{Chiral Constituent Quark Model}

The key to understand the structure of the baryons, in the $\chi \mathrm{CQM}$ formalism [22], is the fluctuation process $q^{ \pm} \rightarrow \mathrm{GB}+q^{\prime \mp} \rightarrow\left(q \bar{q}^{\prime}\right)+q^{\prime}$, where GB represents the Goldstone boson and $q \bar{q}^{\prime}+q^{\prime}$ constitute the "quark sea" $[22,13]$. The effective Lagrangian describing the interaction between quarks and a nonet of GBs is $\mathscr{L}=c_{8} \overline{\mathbf{q}}\left(\Phi+\zeta \frac{\eta^{\prime}}{\sqrt{3}} I\right) \mathbf{q}=c_{8} \overline{\mathbf{q}}\left(\Phi^{\prime}\right) \mathbf{q}$, where $\zeta=c_{1} / c_{8}, c_{1}$ and $c_{8}$ are the coupling constants for the singlet and octet GBs, respectively, $I$ is the $3 \times 3$ identity matrix. The quark spin polarization can be defined as $\Delta q=q^{+}-q^{-}$, where $q^{ \pm}$can be calculated from the spin structure of a baryon $\hat{B} \equiv\langle B|\mathscr{N}| B\rangle=\left\langle B\left|q^{+} q^{-}\right| B\right\rangle$. Here $|B\rangle$ is the baryon wave function and $\mathscr{N}=q^{+} q^{-}$is the number operator measuring the sum of the quark numbers with spin up or down. 
The contributions of the quark sea coming from the fluctuation process can be calculated by substituting for every constituent quark $q^{ \pm} \rightarrow \sum P_{q} q^{ \pm}+\left|\psi\left(q^{ \pm}\right)\right|^{2}$, where the transition probability of the emission of a GB from any of the $q$ quark $\left(\sum P_{q}\right)$ and the transition probability of the $q^{ \pm}$quark $\left(\left|\psi\left(q^{ \pm}\right)\right|^{2}\right)$ can be calculated from the Lagrangian. The general configuration mixing generated by the spin-spin forces has been discussed in the case of octet baryons [23]. The quark polarizations can be calculated from the spin structure of a given baryon as

$$
\hat{B} \equiv\langle B|\mathscr{N}| B\rangle=\cos ^{2} \phi\left\langle 56,0^{+}|\mathscr{N}| 56,0^{+}\right\rangle_{B}+\sin ^{2} \phi\left\langle 70,0^{+}|\mathscr{N}| 70,0^{+}\right\rangle_{B}
$$

\section{Axial Vector Form Factors}

The axial-vector form factors can be expressed in terms of the axial-vector current $A^{\mu, a}$ defined as $\overline{\mathbf{q}} \gamma^{\mu} \gamma_{5} \frac{\lambda^{a}}{2} \mathbf{q}$ through the following matrix elements

$$
\left\langle B\left(p^{\prime}\right)\left|A^{\mu, a}\right| B(p)\right\rangle=\bar{u}\left(p^{\prime}\right)\left[\gamma^{\mu} \gamma_{5} G_{A}^{i}\left(Q^{2}\right)+\frac{q^{\mu}}{2 M_{B}} \gamma_{5} G_{P}^{i}\left(Q^{2}\right)\right] u(p),
$$

where $M_{B}$ is the baryon mass, $u(p)\left(\bar{u}\left(p^{\prime}\right)\right)$ are the Dirac spinors of the initial (final) baryon states, respectively. The four momenta transfer is given as $Q^{2}=-q^{2}$, where $q \equiv p-p^{\prime}$. Here, $\lambda^{a}$ $(a=1,2, . .8)$ are the Gell-Mann matrices of SU(3) describing the flavor structure of the 3 light quarks. It is often convenient to introduce the unit matrix $\lambda^{0}\left(=\sqrt{\frac{2}{3}} I\right)$ in addition to these matrices. In the present context. we shall need only the matrices having diagonal representation corresponding to the flavor singlet current $(a=0)$, isovector current $(a=3)$ and hypercharge axial current $(a=8)$ [24]. The functions $G_{A}^{i}\left(Q^{2}\right)$ and $G_{P}^{i}\left(Q^{2}\right)(i=0,3,8)$ are the axial and induced pseudoscalar form factors respectively. The singlet and non-singlet combinations of the spin structure at zero momentum transfer can be related to the weak couplings and expressed in terms of the spin polarizations

$$
\begin{aligned}
& g_{A, B}^{0}=\left\langle B\left|u^{+} u^{-}+d^{+} d^{-}+s^{+} s^{-}\right| B\right\rangle=\Delta u_{B}+\Delta d_{B}+\Delta s_{B}, \\
& g_{A, B}^{3}=\left\langle B\left|u^{+} u^{-}-d^{+} d^{-}\right| B\right\rangle=\Delta u_{B}-\Delta d_{B}, \\
& g_{A, B}^{8}=\left\langle B\left|u^{+} u^{-}+d^{+} d^{-}+2 s^{+} s^{-}\right| B\right\rangle=\Delta u_{B}+\Delta d_{B}-2 \Delta s_{B} .
\end{aligned}
$$

The $Q^{2}$ dependence of the axial-vector form factors have been experimentally investigated from the quasi elastic neutrino scattering [9] and from the pion electroproduction [10]. The dipole form of parametrization has been conventionally used to analyse the axial-vector form factors

$$
G_{A, B}^{i}\left(Q^{2}\right)=\frac{g_{A, B}^{i}(0)}{\left(1+\frac{Q^{2}}{M_{A}^{2}}\right)^{2}},
$$

where $g_{A}^{0}(0), g_{A}^{3}(0)$ and $g_{A}^{8}(0)$ are the isovector axial-vector coupling constants at zero momentum transfer. For the axial mass $M_{A}$, a global average as extracted from neutrino scattering experiments is $M_{A}=(1.026 \pm 0.021) \mathrm{GeV}$ [25]. Another recent analysis finds a slightly smaller value $M_{A}=(1.001 \pm 0.020) \mathrm{GeV}[26]$. However, in the present work we have used the most recent value obtained by the MiniBooNE Collaboration $M_{A}=1.10_{-0.15}^{+0.13} \mathrm{GeV}$ [27]. 

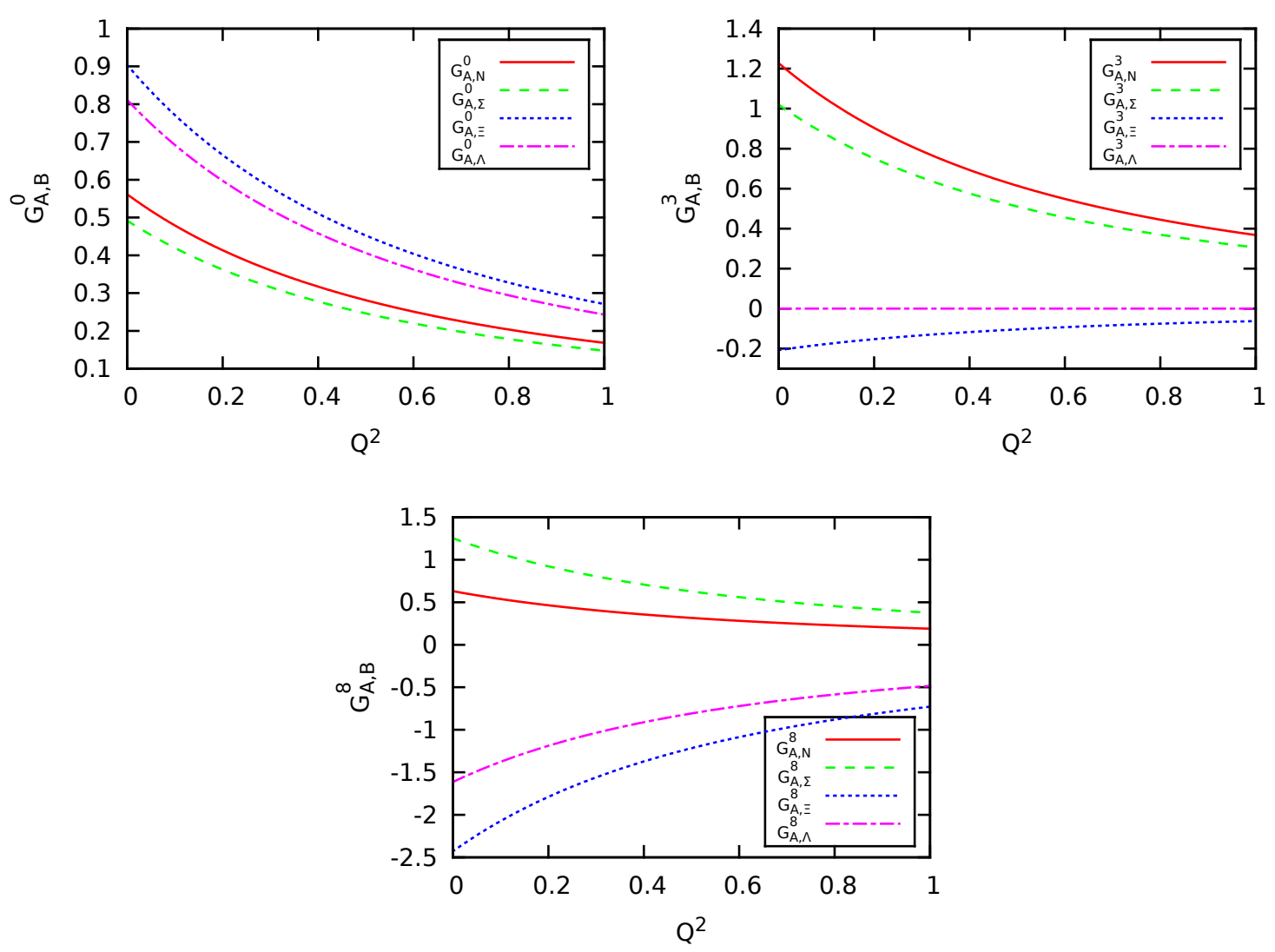

Figure 1: (color online). Form factors for the baryons $N, \Sigma, \Xi, \Lambda$ plotted as function of $Q^{2}$.

After having incorporated $Q^{2}$ dependence in the axial-vector form factors, we now discuss the variation of all the $Q^{2}$ dependent quantities in the range $0 \leq Q^{2} \leq 1$. In Fig. 1, we have presented the singlet and non-singlet axial-vector form factors of the octet baryons $N, \Sigma, \Xi$ and $\Lambda$. The behaviour of the form factors for $\Xi$ and $\Lambda$ is similar to each other. This may possibly due to the presence of more strange quarks in the valence structure. On the other hand, the form factors for $N$ and $\Sigma$, which have the dominance of $u$ quarks in the valence structure, show similar variation with $Q^{2}$. This is true for $G_{A, B}^{0}, G_{A, B}^{3}$ as well as $G_{A, B}^{8}$, however, the case of $G_{A, \Lambda}^{3}$ is different because of its flavor structure which has equal numbers of $u, d$, and $s$ quarks in its valence structure. Unlike the other octet baryons, where the form factors decrease or increase continuously with the $Q^{2}$ values, the form factor in this case has no $Q^{2}$ dependence.

The role of non-valence quarks in the spin structure can be studied in detail by calculating the flavor axial-vector form factors $G_{A, B}^{u}, G_{A, B}^{d}$ and $G_{A, B}^{s}$ using the dipole form of parametrization (Eq. (3.3). In Fig. 2, we have plotted the explicit $u, d$, and $s$ quark flavor contributions for each of the octet baryon axial-vector form factors. The plots clearly project out the valence quark structure of the baryon. For example, since $N$ is dominated by $u$ quark it is clear from the plot of $G_{A, N}^{u, d, s}$ that the $G_{A, N}^{u}$ dominates and $G_{A, N}^{d}, G_{A, N}^{s}$ has a comparatively smaller contribution. The important observation in this case is the non-zero contribution of the $s$ quarks. Even though there are no $s$ quarks in the valence structure the contribution of $G_{A, N}^{s}$ implies a presence of "quark sea" which 

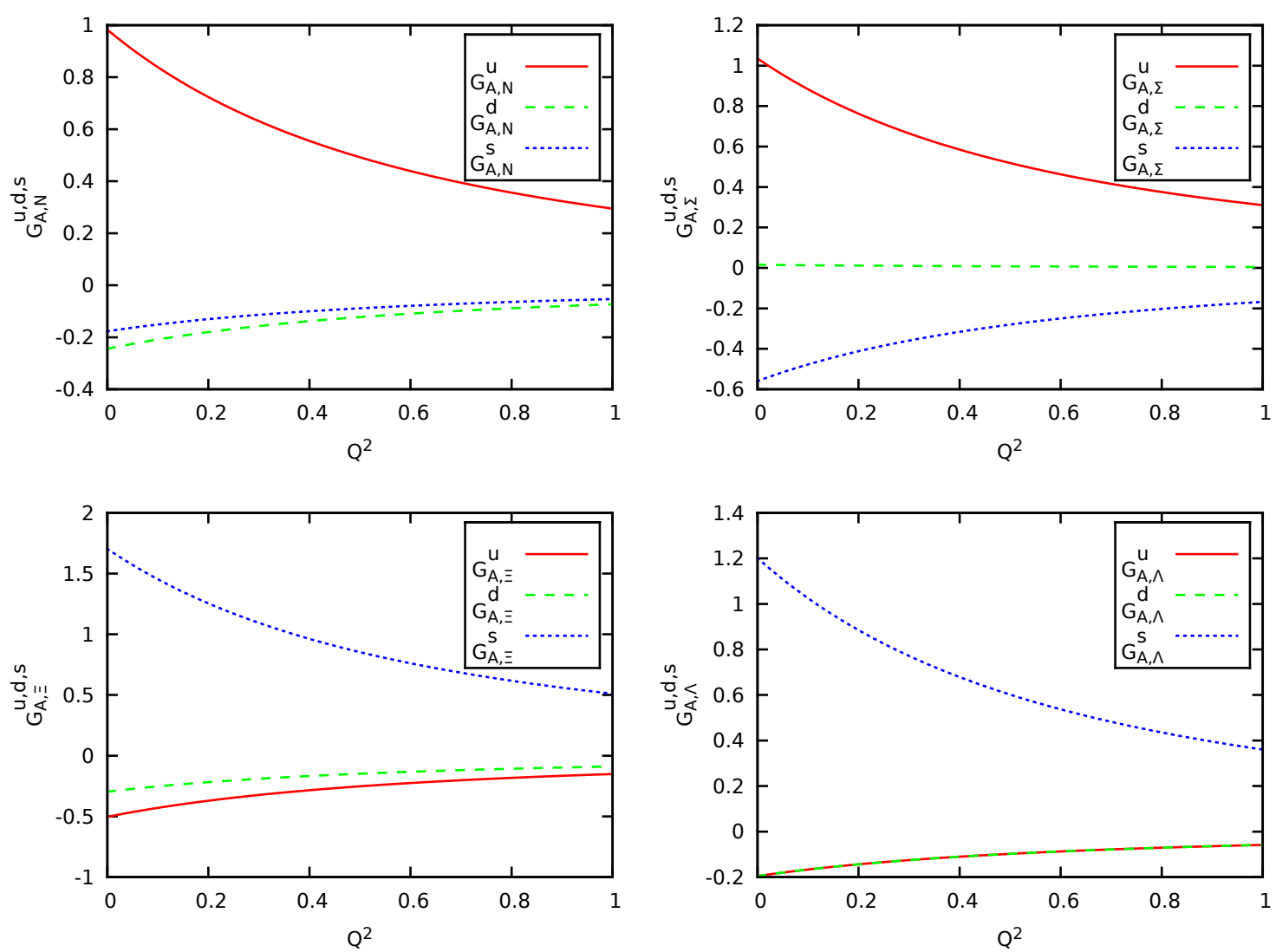

Figure 2: (color online). The explicit flavor form factors for the baryons $N, \Sigma, \Xi, \Lambda$ plotted as function of $Q^{2}$.

is even more at zero momentum transfer. Further, for the case of $G_{A, \Sigma}^{u, d, s}$ and $G_{A, \Xi}^{u, d, s}$, where the valence structure is dominated by the $u$ and $s$ quarks, we find a significant contribution from them. In these form factors, the small but significant $G_{A}^{d}$ can have important implications for the role of sea quarks at low $Q^{2}$. Finally, the $G_{A, \Lambda}^{u, d, s}$, even after having equal contributions from the $u, d$, and $s$ quarks, does not show a symmetric behaviour. The $G_{A, \Lambda}^{s}$ clearly dominates over $G_{A, \Lambda}^{u}$ and $G_{A, \Lambda}^{d}$ which is expected because of the $u$ and $d$ quarks also contribute towards $G_{A, \Lambda}^{u, d, s}$ through quark fluctuations. The future experiments to measure the axial-vector form factors will not only provide a direct method to determine the presence of appropriate amount of quark sea but also impose important constraint on the parity-violating asymmetries in different kinematical regions. Several groups, for example, Minerva are contemplating the possibility of performing the high precision measurements over a wide $Q^{2}$ region in the near future.

\section{References}

[1] J. Ashman et al. (EMC Collaboration), Nucl. Phys. B 328, 1 (1989); B. Adeva et al. (SMC Collaboration), Phys. Rev. D 58, 112001 (1998).

[2] P. Adams et al., Phys. Rev. D 56, 5330 (1997); P.L. Anthony et al. (E142 Collaboration), Phys. Rev. 
Lett. 71, 959 (1993); K. Abe et al. (E143 Collaboration), Phys. Rev. Lett. 76, 587 (1996); K. Abe et al. (E154 Collaboration), Phys. Rev. Lett. 79, 26 (1997).

[3] A. Airapetian et al. (HERMES Collaboration), Phys. Rev. D 71, 012003 (2005).

[4] D.T. Spayde et al. (SAMPLE Collaboration), Phys. Lett. B 583, 79 (2004).

[5] D. Androić et al. (G0 Collaboration), Phys. Rev. Lett. 104, 012001 (2010).

[6] F.E. Maas et al. (PVA4 Collaboration), Phys. Rev. Lett. 94, 152001 (2005).

[7] K.A. Aniol et al. (HAPPEX Collaboration), Eur. Phys. J. A 31, 597 (2007); Z. Ahmed et al. (HAPPEX Collaboration), arXiv:1107.0913v1 [nucl-ex].

[8] K.A. Olive et al. (Particle Data Group), Chin. Phys. C, 38, 090001 (2014).

[9] L.A. Ahrens et al., Phys. Rev. D 35, 785 (1987); L. A. Ahrens et al., Phys. Lett. B 202, 284 (1988).

[10] A. Liesenfeld et al. (A1 Collaboration), Phys. Lett. B bf 468, 20 (1999).

[11] Antonio Silva, Hyun-Chul Kim, Diana Urbano and Klaus Goeke, Phys. Rev. D 72, 094011 (2005); M.R. Schindler and S. Scherer, Eur. Phys. J. A 32, 429 (2007); Guray Erkol and Altug Ozpineci, Phys. Rev. D 83, 114022 (2011); Stephen Pate and Dennis Trujillo, EPJ Web Conf. bf 66, 06018 (2014).

[12] S. Weinberg, Physica A 96, 327 (1979); A. Manohar and H. Georgi, Nucl. Phys. B 234, 189 (1984); E.J. Eichten, I. Hinchliffe, and C. Quigg, Phys. Rev. D 45, 2269 (1992).

[13] H. Dahiya and M. Gupta, Phys. Rev. D 64, 014013 (2001); H. Dahiya and M. Gupta, Phys. Rev. D 66, 051501(R) (2002); H. Dahiya and M. Gupta, Phys. Rev. D 67, 114015 (2003).

[14] H. Dahiya and M. Gupta, Eur. Phys. J. C 52, 571 (2007); N. Sharma and H. Dahiya, Phys. Rev. D 81, 114003 (2010).

[15] N. Sharma, H. Dahiya, P.K. Chatley, and M. Gupta, Phys. Rev. D 79, 077503 (2009); N. Sharma, H. Dahiya, and P.K. Chatley, Eur. Phys. J. A 44, 125 (2010).

[16] H. Dahiya and M. Gupta, Phys. Rev. D 78, 014001 (2008); H. Dahiya and N. Sharma, Mod. Phys. Lett. A, Vol. 26, No. 4, 279 (2011).

[17] N. Sharma, A.M. Torres, K.P. Khemchandani, and H. Dahiya, Eur. Jol. Phys. A 49, 11 (2013).

[18] A.M. Torres, K.P. Khemchandani, N. Sharma, and H. Dahiya, Eur. Jol. Phys. A 48, 185 (2012).

[19] H. Dahiya and M. Gupta, Phys. Rev. D 67, 074001 (2003).

[20] N. Sharma, H. Dahiya, P.K. Chatley, and M. Gupta Phys. Rev. D 81, 073001 (2010).

[21] N. Sharma and H. Dahiya, Int. Jol. of Mod. Phys. A, Vol. 28, No. 14, 1350052 (2013).

[22] T.P. Cheng and L.F. Li, Phys. Rev. Lett. 74, 2872 (1995); Phys. Rev. D 57, 344 (1998); Phys. Rev. Lett. 80, 2789 (1998); J. Linde, T. Ohlsson, and H. Snellman, Phys. Rev. D 57, 452 (1998); 57, 5916 (1998).

[23] A. De Rujula, H. Georgi and S.L. Glashow, Phys. Rev. D 12, 147 (1975); A. Le Yaouanc, L. Oliver, O. Pene and J.C. Raynal, Phys. Rev. D 15, 844 (1977); N. Isgur, G. Karl and R. Koniuk, Phys. Rev. Lett. 41, 1269 (1978); P. Geiger and N. Isgur, ibid. 55, 299 (1997).

[24] D. Barquilla-Cano, A.J. Buchmann, E. Hernandez, Eur. Phys. J. A 27, 365 (2006).

[25] V. Bernard, L. Elouadrhiri and U.-G. MeiÃ§ner, J. Phys. G 28, R1 (2002).

[26] H. Budd, A. Bodek and J. Arrington, Nucl. Phys. Proc. Suppl. 139, 90-95 (2005).

[27] A.A. Aguilar-Arevalo et al. (MiniBooNE Collaboration), Phys. Rev. D 82, 092005 (2010); Tomasz Golan, Krzysztof M. Graczyk, Cezary Juszczak, Jan T. Sobczyk, Phys. Rev. C 88, 024612 (2013). 